\title{
Binary Stars in Praesepe
}

\author{
PAVEL KROUPA \& CHRISTOPHER A. TOUT \\ Institute of Astronomy, Madingley Road, Cambridge CB3 0HA, England
}

\begin{abstract}
In open clusters the scatter about the single-atar main sequence is usually negligible. However, unresolved binary systems are brighter and redder than single stars, and thus some 'stars' appear shifted away from the main sequence. We make very simple models of the Praesepe cluster, and find evidence that low-mass systems prefer independent component masses, whereas systems of higher mass than the sun appear to favour some correlation towards equal-mass components.
\end{abstract}

\section{INTRODUCTION}

There is evidence for correlated component masses among bright binary systems (Eggleton et al. 1989), and evidence for no correlation among faint $\left(M_{\mathrm{V}}>5\right)$ systems (Kroupa et al. 1991). The shape of the stellar luminosity function at magnitudes fainter than $M_{\mathrm{V}} \approx 12$ is very sensitive to the properties of unresolved binary stars (see Kroupa et al. , this Colloquium) but the data are incomplete for the Praesepe cluster.

High quality colour-magnitude data for open clusters offer another opportunity to study the mass-ratio distribution as a function of system mass which does not rely on close inspection of each 'star'. A 'second sequence' $0.75 \mathrm{mag}$ brighter than the single-star main sequence is clearly discernible in a number of Galactic clusters and can result from unresolved binary systems. Johnson (1952) published photo-electric measurements of Praesepe members shown in Figure 1.

We address two specific questions: How large is the proportion of binaries among single stars? And, are the data consistent with the hypothesis that component masses in binaries are uncorrelated? A more detailed discussion is to be found in Kroupa (1992).

\section{MODEL BASIS}

We make Monte-Carlo simulations of the data by generating 100,000 systems. We use a mass function for stars given by Kroupa et al. (these proceedings) and use their main-sequence mass-luminosity relation to assign each mass an absolute visual magnitude. We assume that all cluster members have the same composition, distance $(167 \mathrm{pc})$ and age $\left(8 \times 10^{\mathbf{8}} \mathrm{yrs}\right)$. Pre- and post-main-sequence evolution need not be taken into account for stars with $8.4<V<14$. We assume that a fraction $f$ of all systems are unresolved binary stars.

\section{BINARY SYSTEMS AND THE CMD}

Estimates of the binary fraction are usually based on the number, $n_{\mathrm{b}}$, of 'stars' about $0.2 \mathrm{mag}$ brighter than the main sequence compared with the number, $n_{\mathrm{m}}$, within $0.2 \mathrm{mag}$ of the main sequence. Assuming the photometric binary fraction to be given by $f_{\mathrm{p}}=n_{\mathrm{b}} / n_{\mathrm{m}}$ these approaches typically yield $f_{\mathrm{p}} \approx 0.25$. 


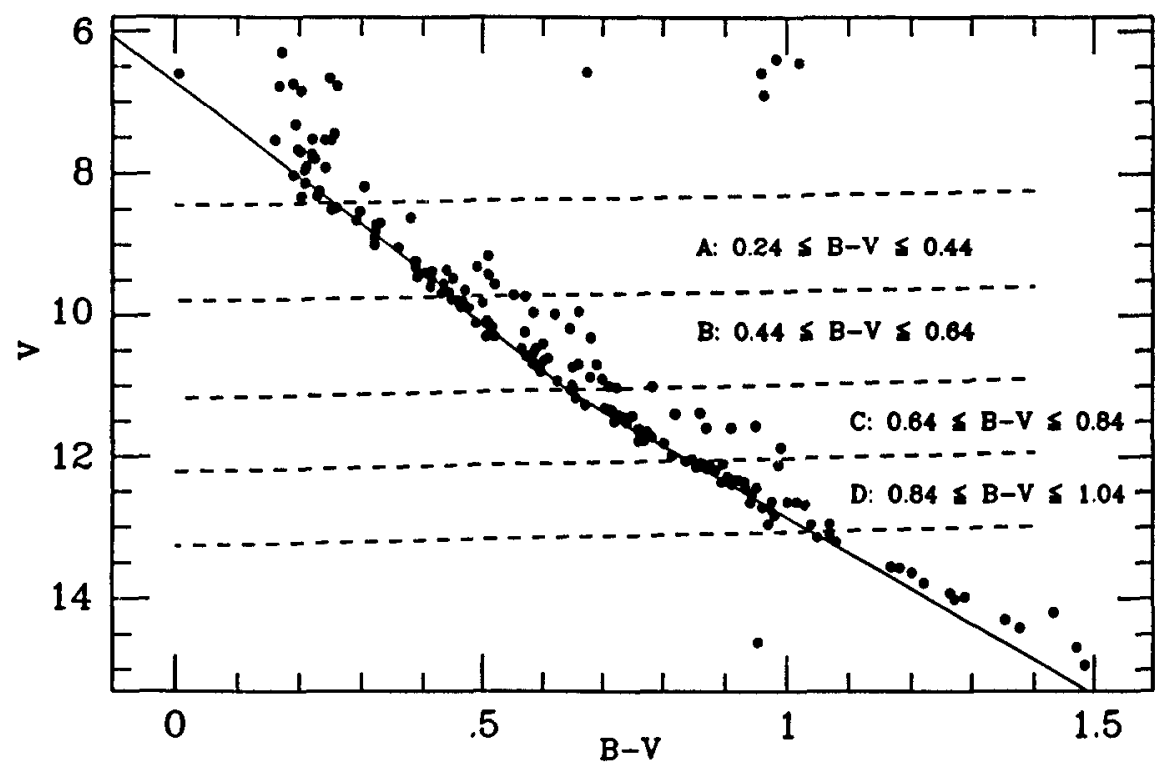

FIGURE 1. Colour-magnitude diagram for Praesepe. The fiducial main sequence is shown by the solid line. Boundaries of the four regions used in Section 4 are indicated by the near-horizontal lines which would be perpendicular to the fiducial main sequence if both axes had the same scale.

To study this we transform the $V$-band to the Cousins $I$-band using a fiducial main sequence. A second sequence arises quite naturally from our assumption that component masses are uncorrelated but is not as pronounced as in Figure 1. We count the number of objects with $9<V<14$ and $0.2 \mathrm{mag}$ nitudes brighter than the main sequence. For our models with $f=0.4$ we find $f_{\mathrm{p}}=0.10$ and with $f=0.8, f_{\mathrm{p}}=0.19$. Applying a similar procedure to Praesepe we obtain $f_{\mathrm{p}}=0.24$ with $n_{\mathrm{b}}=17$. Bolte (1991) finds that at least 10 out of these 17 'stars' are spectroscopic binaries. Our simulation with $f=1$ gives $f_{\mathrm{p}}=0.24$ but the Poisson error alone is 0.06 . These simulations suggest that the true proportion of binary systems in the Praesepe may be as large as $100 \%$ if the companion masses are not correlated.

\section{CORRELATED MASSES}

We introduce the parameter $f_{1}$ as the proportion of binary systems in which the masses are uncorrelated and chosen from the same mass function. Above we assumed $f_{1}=1$. Here we take $f_{1} \leq 1$ and assume that the remaining fraction, $1-f_{1}$, of binary systems consist of two equal-mass stars. The absolute $B-$ band magnitude is obtained from our fiducial colour-magnitude relation, $M_{\mathbf{V}}=$ $a(B-V)+b$, where $a$ and $b$ are chosen so as to fit the main sequence for single stars in Figure 1. We subdivide the $V, B-V$ plane into four colour ranges as shown in Figure 1 and find solutions in $f, f_{1}$ space for each. Regions A, B and $\mathrm{C}$ lead to very similar solutions which differ from the solution in Region D. Our results imply an enhanced preference for equal-mass binary components for $B-V<0.8$, as shown in Figure 2. 


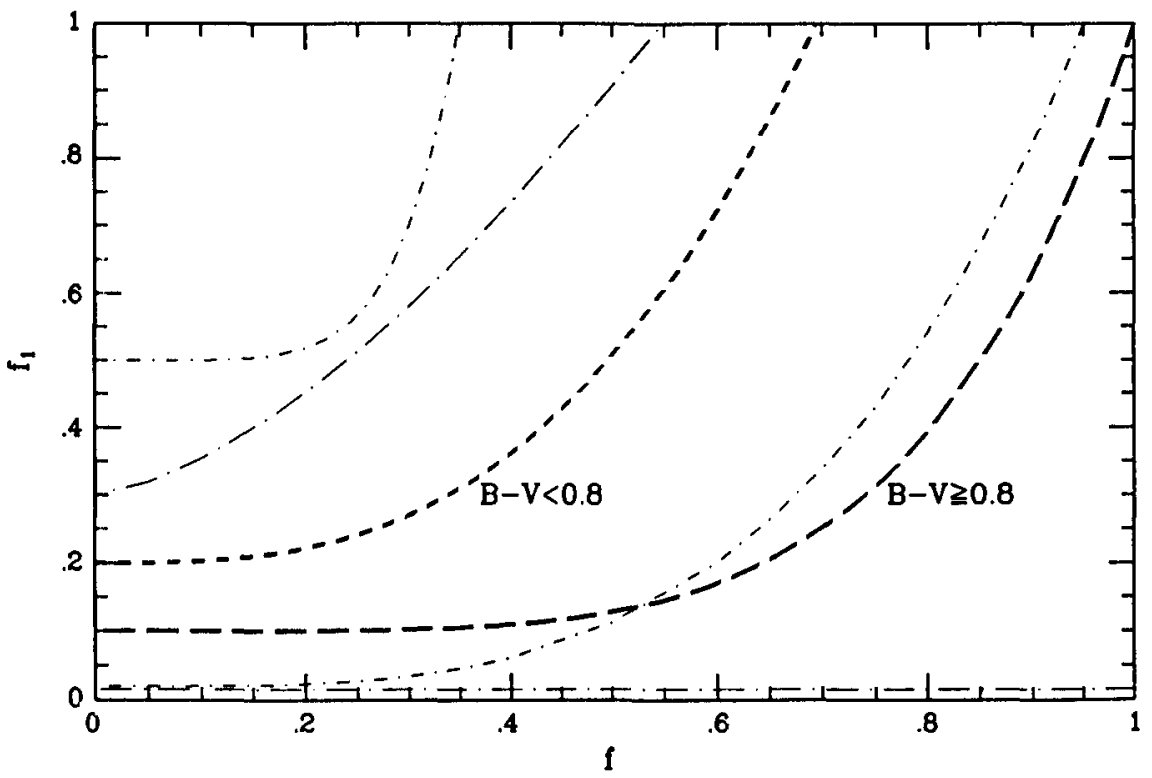

FIGURE 2. (Based on Figure 6.5 of Kroupa 1992). Required values of the binary fraction, $f$, and the fraction of these which are uncorrelated, $f_{1}$, are shown for regions $A$ to $D$. In each case the significance increases to a maximum of about $80 \%$ as $f$ rises to unity. The $5 \%$ confidence contours are given approximately by the long-dashed-dotted lines for $B-V \geq 0.8$ and by the short-dash-dotted lines for $B-V<0.8$.

\section{CONCLUSIONS}

1. High-quality colour-magnitude data for open clusters can be used to constrain the relative proportion of binaries among all 'stars'.

2. We find solutions with a proportion close to unity though smaller fractions are not excluded.

3. Systems with combined colour redder than $B-V \approx 0.8$ appear to prefer uncorrelated component masses whereas at bluer colours the proportion of equal-mass systems among binaries increases to $30 \%$ if all 'stars' are assumed to be unresolved binaries.

These results together with those of Kroupa et al. (1991) and Eggleton et al. (1989) suggest that the mass-ratio distribution may change character around $B-V \approx 0.8\left(\mathcal{M} \approx 0.8 \mathcal{M}_{\odot}\right)$.

\section{REFERENCES}

Bolte, M. 1991, ApJ, 376, 514

Eggleton, P. P., Fitchett, M. J., \& Tout, C. A. 1989, ApJ, 347, 998 Johnson, H. L. 1952, $A p J, 116,640$

Kroupa, P., Tout, C. A., \& Gilmore, G. 1991, MNRAS, 251, 293

Kroupa, P. 1992, Ph.D. thesis, Cambridge University 